\title{
BACKGROUND: OBESITY AS A PUBLIC HEALTH PROBLEM
}

Obesity is defined as a body mass index (BMI) of $30 \mathrm{~kg} / \mathrm{m}^{2}$ or more, where a person's BMI is defined as their weight in $\mathrm{kg}$ divided by the square of their height in metres. Overweight is defined as a BMI between 25 and $29.9 \mathrm{~kg} / \mathrm{m}^{2}$. In 1980, $6 \%$ of men and $8 \%$ of women in the UK were obese. In 2000, the respective figures had increased to $21 \%$ and $21.4 \%$. Approximately $55 \%$ of the adult population is overweight or obese. ${ }^{1}$

Obesity causes or exacerbates a large number of health problems, both independently and in association with other diseases. ${ }^{2}$ In particular, it is associated with the development of type 2 diabetes mellitus, coronary heart disease, an increased incidence of certain forms of cancer, obstructive sleep apnoea, and osteoarthritis of large and small joints. The Build and Blood Pressure Study has shown that the adverse effects of excess weight tend to be delayed, sometimes for 10 years or longer. ${ }^{3}$ Life insurance data and epidemiological studies confirm that increasing degrees of overweight and obesity are important predictors of decreased longevity. In the Framingham Heart Study, the risk of death within 26 years increased by $1 \%$ for each extra pound $(0.45 \mathrm{~kg})$ increase in weight between the ages of 30 years and 42 years, and by $2 \%$ between the ages of 50 years and 62 years. ${ }^{4}$ Despite this evidence, many clinicians continue to consider obesity to be a self-inflicted condition of little medical significance.

Increasing body fatness is accompanied by profound changes in physiological function. These changes are, to a certain extent, dependent on the regional distribution of adipose tissue. Generalised obesity results in alterations in total blood volume and cardiac function while the distribution of fat around the thoracic cage and abdomen restricts respiratory excursion and alters respiratory function. The intra-abdominal visceral deposition of adipose tissue, which characterises upper body obesity, is a major contributor to the development of hypertension, elevated plasma insulin concentrations and insulin resistance, hyperglycaemia, and hyperlipidaemia.

In 1988, Reaven coined the term syndrome X to refer to the clustering of (abdominal) obesity, hypertriglyceridaemia, reduced levels of high density lipoprotein (HDL) cholesterol, hyperinsulinaemia, glucose intolerance, and hypertension. ${ }^{5}$ To this basic cluster of abnormalities have been added further metabolic alterations that include increased atherogenic small, dense low density lipoprotein particles, elevated apo B concentrations, and raised plasminogen activator inhibitor 1 . The syndrome is now referred to as the metabolic syndrome or insulin resistance syndrome, the latter identifying the likely pivotal biochemical abnormality. Reaven estimated that the prevalence of insulin resistance within the sedentary adult population of North America is approximately $25 \%$ and closely linked to central (visceral) obesity. Several cohort studies have confirmed that upper body (visceral) obesity is associated with greater cardiovascular morbidity and mortality than obesity itself.

Obesity is not a single disorder but a heterogeneous group of conditions with multiple causes. Although genetic differences are of undoubted importance, the marked rise in the prevalence of obesity during the past 30 years is best explained by behavioural and environmental changes that have resulted from technological advances. An effective programme for the management of overweight and obesity must take account of these changes and address the aetiological factors

See end of article for authors' contributing to weight gain.

\section{AETIOLOGY OF HUMAN OBESITY}

Correspondence to:

Professor P Kopelman, Queen Mary's School of Medicine and Dentistry, Turner St, London El 2AD, UK p.g.kopelman@qmul.ac.uk
At its simplest level the "cause" of obesity is already known. By definition it results from an imbalance between energy intake and energy expenditure. In any individual excessive caloric intake or low energy expenditure, or both, may explain the development of obesity. Multiple studies over several decades have attempted to clarify the relative roles of energy intake and energy expenditure in human obesity. The lack of consensus, despite intensive efforts, reflects a 
number of problems inherent to the study of the obese state. These include the heterogeneity of human obesity, the imprecision of measurements of energy intake and expenditure, combined with the minor degrees of energy imbalance required to accumulate fat tissue over time.

\section{Role of altered energy intake in the pathogenesis of obesity}

It is surprising that no direct correlation has been reported between increasing obesity prevalence and increased energy intake in developed nations given the ready availability of highly palatable foods. However, the lack of a direct correlation may be explained by underreporting of food intake that confounds the understanding of the role of energy intake in the aetiology of obesity. Underreporting is widely recognised as a feature of obesity with comparisons of energy intake and expenditure in obese subject showing a consistent shortfall in self-reported food intake of approximately $30 \%$ of the energy requirements. ${ }^{6}$ It is thus extremely difficult to obtain reliable data on the energy intake of obese subjects and this makes the interpretation of eating surveys in the UK suggesting a decline in total calorie ingestion difficult. Proxy measures of energy intake do suggest altered intakes may have occurred since the 1970s: in the USA, documented portion sizes have increased $2-5$-fold. ${ }^{7}$

\section{Role of altered energy expenditure in the development and maintenance of obesity}

In developed countries there is a relationship between low levels of physical activity and obesity. A longitudinal Finnish study found that those reporting physical exercise three or more times each week had on average lost weight since a preceding survey. By contrast, those who undertook little physical activity gained weight and had twice the risk of gaining $5 \mathrm{~kg}$ or more. ${ }^{8}$ Among children in the USA, the relative risk of obesity is 5.3 times greater for children who watch television for five hours or more each day compared with those children who watch for less than two hours, even after correcting for a wide range of socioeconomic variables.

In the UK, health surveys indicate a reduction in daily calorie intake across the population. ${ }^{10}$ Nevertheless, a study combining data on energy intake and physical activity in relation to the secular increase in adult obesity shows a close relationship between proxy measures of physical activity (television viewing and car ownership) and the prevalence of obesity. ${ }^{11}$

\section{REGULATION OF ENERGY INTAKE}

Food intake may be restricted either by a conscious decision to eat less (dietary treatment) or through pharmacologically "imposed" alterations in the central regulation of appetite control. Alternatively, absorption of nutrients can be artificially reduced either by drug inhibition of digestive processes or surgically induced anatomical alterations to bowel anatomy. New thoughts on managing obesity are beginning to build on the better understanding of these principles.

Eating is dependent on a large number of factors that include availability of food, an individual's mood, and the physical attributes of the food such as appearance, smell, and palatability. The latter is now a complex science as a result of food technology. Food consumption in humans is regulated through a number of complex biological mechanisms designed to ensure that body weight remains relatively constant over long periods.
NEUROREGULATION OF APPETITE AND EATING

The original theories identified two discrete localised areas in the hypothalamus that regulated eating: lesions of the ventromedial hypothalamic nucleus (VMN) in rodents resulted in voracious eating and obesity whereas lesions of the ventrolateral hypothalamic nucleus led to starvation and death. The existence of a circulating factor that influenced satiety was raised by the results of parabiosis (cross circulation) experiments. Parabiosis of normal and obese VMN lesioned rats resulted in inhibition of feeding and weight loss in normal weight animals. In contrast, VMN lesioned rats were unaffected suggesting that their hypothalami lacked the ability to respond to a presumed circulating satiety factor. Identification of leptin and the discovery of its synthesis and secretion by adipocytes suggested this as the circulating satiety factor. ${ }^{12}$ Administration of leptin to rodents genetically deficient in its synthesis $(o b / o b)$ reverses the hyperphagia, obesity, and neuroendocrine abnormalities. In contrast, no benefit is seen in animals that lacked functional leptin receptors ( $d b / d b$ rodents).

\section{Interaction between circulating factors and neuronal pathways in the hypothalamus}

Leptin is synthesised mainly in adipose tissue although low levels of expression have been detected in many tissues, including skeletal muscle, gastric fundus, and placenta. Leptin circulates as free and bound hormone and reaches its targets in the brain via a saturable process. The importance of leptin to energy balance is evident in humans where rare mutations of leptin or leptin receptor genes result in increased appetite, morbid obesity, and associated metabolic and endocrine abnormalities. ${ }^{13}$ Plasma leptin concentration in obesity increases in proportion to body fat such that plasma levels are higher in obese than in lean weight people. ${ }^{14}$

Leptin affects several aspects of energy balance. In rodent models of obesity, a reduction in leptin during fasting partly accounts for stimulation of appetite, activation of the hypothalamic-pituitary axis, and suppression of thyroid and reproductive hormones. By contrast, a rise in leptin levels decreases meal size and modulates taste perception. Leptin mediates these actions through its action within hypothalamic nuclei. ${ }^{15}$ Leptin inhibits the synthesis and release of neuropeptide Y (NPY) and agouti related peptide (ARGP) and increases pro-opiomelanocortin (POMC). POMC is a precursor of alpha melanocyte stimulating hormone ( $\alpha$-MSH) and cocaine and amphetamine regulated transcript (CART) through a direct action on synthesis in the arcuate nucleus. The anorexic action of $\alpha$-MSH is regulated through melanocortin receptor 3 and 4 (MC3R, MC4R). NPY/AGRP and $\alpha$-MSH/CART neurones project to the paraventricular hypothalamic nuclei (PVN) and lateral hypothalamic area (LHA). Leptin additionally regulates feeding indirectly by suppressing the release of melanin concentrating hormone $(\mathrm{MCH})$ and orexin in the LHA. The VMH is additionally regulated directly by leptin.

Figure 1 illustrates the postulated actions of leptin and the related peptides on appetite regulation.

Antagonism of anorexigenic (appetite suppressing) melanocortin signals is caused by orexigenic (appetite stimulating) peptides such as ARGP and NY, which are coexpressed. AGRP antagonises the interaction between $\alpha$-MSH and MC4R, and NPY decreases expression of the gene encoding 


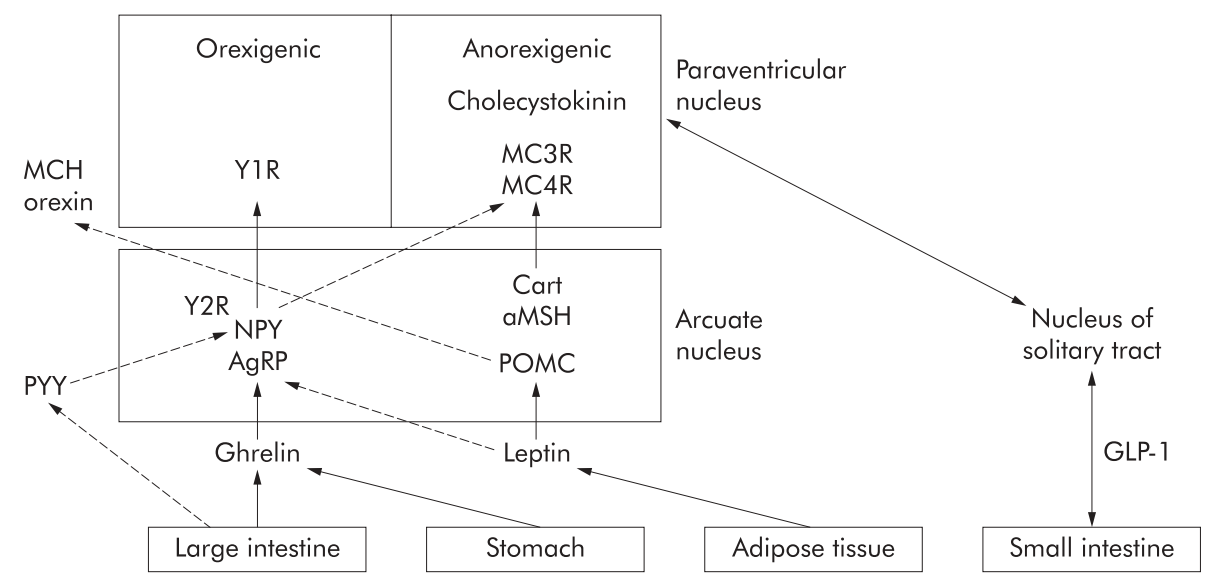

Figure 1 A diagrammatic representation of the complex interrelationship between peripheral and central (hypothalamic) neuropeptides for regulation of feeding. Stimulation by orexigenic pathways results in food intake; stimulation of anorexigenic pathways leads to satiety. Continuous lines represent stimulating pathways, broken lines indicate suppressive actions. NPY, neuropeptide Y; AgRP, argouti related peptide; POMC, proopiomelanocortin; PYY, peptide YY; Y21, Y2R, postsynaptic NPY receptors; Cart, cocaine amphetamine regulated transcript; MC3R, MC4R, melanocortin receptor 3 and $4 ; M C H$, melanin concentrating hormone; GLP-1, glucagon-like peptide.

POMC. NPY also decreases the synthesis of thyrotropin releasing hormone and increases the synthesis of $\mathrm{MCH}$.

\section{Monoaminergic pathways and appetite regulation}

The suppressive effect of monoamines on feeding is well recognised. Serotonin is synthesised in the dorsal raphe nucleus and distributed to the PVN, the VMN, and other forebrain regions. Serotonin inhibits food intake and reduces body weight in rodents by suppressing appetite and increasing energy expenditure. Mice with targeted disruption of the $5-\mathrm{HT}_{2 \mathrm{c}}$ receptor develop hyperphagia, obesity, and diabetes, suggesting that the antiobesity action of serotonin is mediated by this receptor subtype. ${ }^{16}$

\section{PERIPHERAL SIGNALS MEDIATING THE CENTRAL REGULATION OF FOOD INTAKE}

Signals that provide short term information about hunger and satiety include Glucagon-like peptides (GLPs), cholecystokinin, ghrelin, and peptide $\mathrm{YY}_{3-36}$ (PYY). ${ }^{17}$ The release of cholecystokinin from the upper gut and the release of GLP-1 and PYY more distally provide mechanical regulation mediated through the ileal braking mechanism. There are, additionally, signals from vagal afferent neurones within the gastrointestinal tract that respond to mechanical deformation, macronutrients, $\mathrm{pH}$, tonicity, and hormones. Neural and humoral signals are then integrated in specific regions of the hypothalamus and brain stem

GLP-1 and GLIP-2 are produced by L cells in the intestine and secreted in response to nutrients. GLP-1 reduces plasma glucose through multiple effects on gastric emptying, glucose dependent insulin release, synthesis and release of insulin, and glucagons. ${ }^{18}{ }^{19}$ In the central nervous system, GLP-1 is produced in the nucleus tractus solitarius and distributed to the PVN and other regions within the forebrain (fig 1). In humans, GLP-1 appears to decrease glucose and appetite. Various other gut hormones have been implicated in satiety-cholecystokinin, bombesin, gastric inhibitory peptide, enterostatin, and PYY. These peptides appear to act synergistically with gastrointestinal distension to reduce meal size. Cholecystokinin is also expressed locally in the lateral parabrachial nucleus and PVN and inhibits eating after central administration in animals.
PYY is produced by the intestine, in proportion to the calories ingested in a meal, and inhibits eating for up to 12 hours by an action on the arcuate nucleus. It binds the postsynaptic Y2 receptor on NPY neurones to inhibit NPY/ AGRP and ultimately food intake. ${ }^{20}$

Ghrelin is an acylated 28 amino acid peptide produced mainly by the stomach. and appears to be intimately involved in energy balance. Circulating levels of ghrelin are increased during fasting and cachexia and decreased in response to obesity and acute food ingestion. ${ }^{21}$ In rodents, ghrelin increases body weight by stimulating food intake and reducing fat oxidation. Ghrelin containing neurones have been detected in the ventrobasal hypothalamus suggesting that the effect of ghrelin on feeding is mediated locally in the brain. This is consistent with the ability of ghrelin to stimulate NPY/AGRP mRNA expression.

\section{MANAGING OBESITY}

The management of overweight and obesity is directed primarily to reduce energy intake and increase energy expenditure. There are numerous strategies that can be used to induce negative energy balance and short term weight loss. However, due to the chronic and relapsing nature of obesity, it is the long term efficacy of these approaches on maintaining lowered weight (and minimising the risk of related chronic diseases) that is of fundamental importance (see box 1).

\section{LIFESTYLE MODIFICATION Dietary treatment}

Traditionally, low calorie diets (800-1500 kcal/day) that incorporate various methods for restricting intake have been recommended for weight management. The use of low calorie diets with a treatment period beyond six months has been associated with a mean weight loss of $\sim 8 \%$ although with longer use (3-4.5 years) this is reduced to $\sim 4 \%{ }^{22}$ Generally,

\section{Box 1}

Obesity management $=$ weight loss + weight maintenance + risk reduction 
these strategies aim to provide a macronutrient composition of $30-35 \%$ energy from fat, $50 \%$ from total carbohydrate, and $10-15 \%$ from protein in line with national dietary guidelines. ${ }^{23}$ There is considerable debate as to the ideal macronutrient composition for weight management and risk reduction, and clarification will only occur with a larger number of well designed randomised controlled trials. It is increasingly recognised that no one dietary treatment will be suitable for all individuals and there is a need to match treatments to a patient's requirements and preferences (box 2).

\section{Low fat high carbohydrate diets}

Low fat ( $\leqslant 30 \%$ energy from fat) high carbohydrate diets play a central role in the dietary management of overweight and obesity. To date, three meta-analyses have examined the effect of reducing fat intake (ad libitum diets) on weight in lean, overweight, and obese subjects. Fat restriction seems an effective method of lowering energy density and is associated with spontaneous weight loss. ${ }^{24-26}$ A $10 \%$ reduction in dietary fat leads to $\sim 3-4 \mathrm{~kg}$ weight loss in normal-overweight subjects and $\sim 5-6 \mathrm{~kg}$ weight loss in the obese. ${ }^{25}$ However, what remains unclear is the relative effectiveness of low fat ad libitum diets compared with low fat energy restricted approaches. ${ }^{27}$ The feasibility of sustaining modest weight loss ( 3-4 kg) over time using a low fat high carbohydrate diet as part of a comprehensive treatment package has been demonstrated in several studies with an accompanying risk reduction for type 2 diabetes of $58 \% .^{28}{ }^{29}$ It is the influence of low fat diets and the modification of fatty acid composition that is important for the reduction of risk for coronary heart disease.

\section{Fixed energy deficit diets}

This approach has become increasingly popular and is commonly used by dietitians to provide a structured eating plan based on estimated energy requirements. Individual energy requirements are estimated by calculating basal metabolism, adjusting for physical activity and subtracting an energy deficit $(\sim 600 \mathrm{kcal} /$ day $)$ to induce $\sim 0.5 \mathrm{~kg} /$ week weight loss. The practice of advising a fixed energy allowance (for example, $1200 \mathrm{kcal} / \mathrm{day}$ ) to all individuals regardless of severity of obesity has been questioned. The greater the obesity the higher the energy requirements and the larger the energy deficit imposed. There is some evidence to suggest that modest energy reductions ( $\sim 600 \mathrm{kcal} /$ day) may improve compliance $^{30}$ and this approach has been recommended as a dietary option for weight management. ${ }^{31}$

\section{Meal replacements}

Meal replacement programmes are controlled portion products (shakes, bars, soups, and pastas) that replace two meals (and snacks) per day while allowing inclusion of one regular meal of "healthy" foods. This approach generally provides $1200-1600 \mathrm{kcal} /$ day. A recent meta-analysis

Box 2

Key dietary messages for managing weight
Low fat, particularly saturated fat
Increase wholegrain high fibre carbohydrates
Increase fruit and vegetables
Consider portion sizes

summarised the efficacy of the approach compared with conventional energy restricted diets. In the short term (three months), weight loss was higher in the group treated with meal replacements compared with the "standard" intervention group and importantly this difference was still evident at one year. ${ }^{32}$ However, the efficacy of meal replacements in real life settings where meal replacement products need to be purchased, and may be discontinued at an early stage, has not been investigated.

\section{Very low calorie diets}

Very low calorie diets (VLCDs) are formula foods providing in the region of $450-800 \mathrm{kcal}$ per day, used as the single source of nutrition and replacing all meals and snacks. Diets providing such low energy intakes are often associated with a feeling of fatigue, constipation, nausea, and occasionally diarrhoea. A more serious side effect is the development of gallstones associated with the rapid weight loss ( $1-2 \mathrm{~kg} /$ week). Due to the potential adverse effects of these diets, they are generally reserved for patients with severe obesity (BMI $35 \mathrm{~kg} / \mathrm{m}^{2}$ or greater) and associated comorbidities that justify rapid weight loss. Evidence suggests VLCDs are no more effective in long term management than more moderate dietary strategies. ${ }^{22}$ This is despite combining VLCDs with a behavioural approach and/or pharmacotherapy. ${ }^{33}$

\section{Low glycaemic index diets}

The glycaemic index (GI) describes the effect of carbohydrate foods on postprandial glycaemia and is measured by comparing the glycaemic effect of the test food with that of a reference food such as glucose or white bread. Low GI foods are more slowly absorbed leading to a less acute effect on glucose and insulin levels, with suggestions this may reduce hunger, increase satiation, and reduce energy intake. However, as no long term randomised controlled trials have evaluated low GI diet in obesity management, it is premature to specifically recommend low GI diets for weight management. ${ }^{34}$ In contrast, epidemiological and interventional evidence does support the value of low GI diets in reducing the risk of coronary heart disease and diabetes, probably by raising HDL cholesterol, suppressing triglyceride levels, and decreasing insulin resistance. ${ }^{35}$ It is appropriate to promote certain constituents of a low GI diet (increased wholegrain cereals, fruit and legume consumption) as part of a conventional energy restricted diet for the long term management of obesity.

\section{High protein low carbohydrate diets}

High protein low carbohydrate diets, such as the Atkins diet, are currently a popular choice for managing weight. However, their popularity defies evidence supporting efficacy and long term safety. Typically, the diet contains a high proportion of protein foods, unrestricted use of fats, particularly saturated fats, and a severe restriction of carbohydrates (refined and complex). Initially, carbohydrate intake is extremely limited ( $20 \mathrm{~g} /$ day) but is subsequently increased by $5 \mathrm{~g}$ /day although intake generally remains below recommended levels. A calculation of the diet's composition suggests it provides on average $27 \%$ energy from protein, $5 \%$ from carbohydrates, and $68 \%$ fat, in sharp contrast with current dietary recommendations. ${ }^{36}$

A key question is whether low carbohydrate high protein diets lead to effective long term weight loss. Insufficient evidence is currently available on which to make such a 
judgement. Two recent studies have suggested that weight loss over the first three to six months ${ }^{37}{ }^{38}$ was higher in the low carbohydrate high protein group. However, this difference was no longer evident at one year, indicating a greater weight regain in the high protein group. ${ }^{38}$ Both studies were of a short duration with limited sample sizes and high attrition rates.

There are a number of safety concerns about the long term use of such diets. The American Heart Association ${ }^{36}$ and the British Dietetic Association (www.bda.co.uk) have issued statements warning against the adoption of this approach. The possible effect of high protein intakes on renal and liver function, particularly in those at elevated risk of developing kidney stones or other renal abnormalities, is unclear. High protein diets may increase calcium excretion and influence bone health. There are fears that the high fat and saturated fat intake, inherent in such diets, have the potential to adversely influence lipid profile, particularly as other protective foods (fruits, wholegrain cereals) are restricted. There is a need to investigate the safety and efficacy of high protein low carbohydrate diets with large randomised controlled trials over an extended period of time.

\section{Evidence for effectiveness of dietary approaches in the management of obesity}

Table 1 summarises the evidence for the effectiveness of the various dietary approaches for managing overweight and obesity.

\section{Physical activity}

The most variable component of energy expenditure is physical activity, representing $20-50 \%$ of total energy expenditure, $^{39}$ and an evaluation of an individual's activity behaviour is critical to any therapeutic assessment. When physical activity alone is used in the treatment of obesity, weight losses are modest and average $2-3 \mathrm{~kg} .{ }^{22}$ For any given weight loss, fat free mass (FFM) is better preserved in exercising than non-exercising subjects: this is important because FFM is the best predictor of resting metabolic rate which is the largest contributor to daily energy expenditure. ${ }^{40}$ Regular physical activity has other important physiological benefits, independent of weight loss, that include reducing blood pressure, improving atherogenic lipid profiles, and improving glucose tolerance. Such benefits are substantial and should be emphasised to all patients.
Physical activity seems to play a critical role in maintaining lost weight with a positive association between the level of activity and the degree of sustained weight loss. This may be explained by additional energy expended, improved maintenance of lean tissue, or the positive psychological influence of activity better equipping the individual to cope with continued behaviour change.

Physical activity recommendations suggest 30 minutes of moderate activity on at least five days of the week. This level of activity is associated with improved fitness and protection from cardiovascular diseases. However, recent evidence has highlighted a longer duration of daily activity required to maintain lowered weight and prevent weight regain, and suggests 45-60 minutes/day may be required (box 3). ${ }^{41}$

\section{Behavioural management}

Behavioural approaches aim to help subjects to implement and sustain changes to their eating and activity behaviour and require trained health professionals with good interpersonal skills to use the approach appropriately. Typical strategies include self-monitoring, realistic goal setting, stimulus control, problem solving, relapse prevention, and stress management. There is strong evidence that combining a behavioural approach with more traditional dietary and activity advice leads to improved short term weight loss and is currently the most effective lifestyle approach to managing weight. ${ }^{42}$ This is supported by the promising longer term data from the Diabetes Prevention Programme in which well trained professionals used a behavioural approach to convey and support eating and activity changes. $^{28}$

\section{PHARMACOLOGICAL TREATMENT OF OBESITY}

There are currently two categories of antiobesity drugs: those that act on the gastrointestinal system (pancreatic lipase inhibitors) and those that act on the central nervous system to primarily suppress appetite. Guidance has been published

\section{Box 3}

Current physical activity recommendations: For adults, 30 minutes of moderately intensive activity (for example, brisk walking) on at least five days of the week.

Table 1 Summary of evidence for various dietary approaches in the management of overweight and obesity

\begin{tabular}{|c|c|c|}
\hline Dietary approach & Evidence of effectiveness & Inconclusive/inadequate evidence \\
\hline \multirow[t]{3}{*}{ Low fat } & Spontaneous weight loss ${ }^{19-21}$ & $\begin{array}{l}\text { Unclear if energy restriction + fat restriction more effective than } \\
\text { fat restriction alone }{ }^{22}\end{array}$ \\
\hline & Risk reduction ${ }^{21}$ & \\
\hline & $\begin{array}{l}\text { Maintenance of modest weight loss with low fat diet as part of } \\
\text { comprehensive treatment programme }{ }^{23}\end{array}$ & \\
\hline Fixed energy deficit & Modest weight loss ${ }^{25}$ & $\begin{array}{l}\text { Some evidence of improved compliance relative to more severe } \\
\text { energy restrictions } \mathrm{s}^{25}\end{array}$ \\
\hline Meal replacements & Weight loss and maintenance ${ }^{27}$ & $\begin{array}{l}\text { Unclear of outcome if product not free } \\
\text { Unclear of effectiveness in morbidly obese }\end{array}$ \\
\hline VLCDs & Short term weight loss greater than $\mathrm{LCD}^{22}$ & 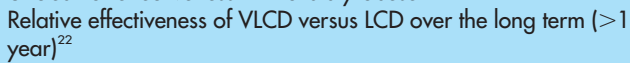 \\
\hline $\begin{array}{l}\text { Low glycaemic index } \\
\text { High protein low carbohydrate }\end{array}$ & Risk reduction for CHD and type 2 diabetes $^{30}$ & $\begin{array}{l}\text { No RCT of low GI diet on weight loss over time }{ }^{29} \\
\text { Short term and long term safety profile unclear }{ }^{31} \\
\text { Effectiveness on long term weight loss and maintenance not } \\
\text { available }\end{array}$ \\
\hline
\end{tabular}

$V L C D$, very low calorie diet; $L C D$, low calorie diet; $C H D$, coronary heart disease, $R C T$, randomised controlled trial, $G I$, glycaemic index. 


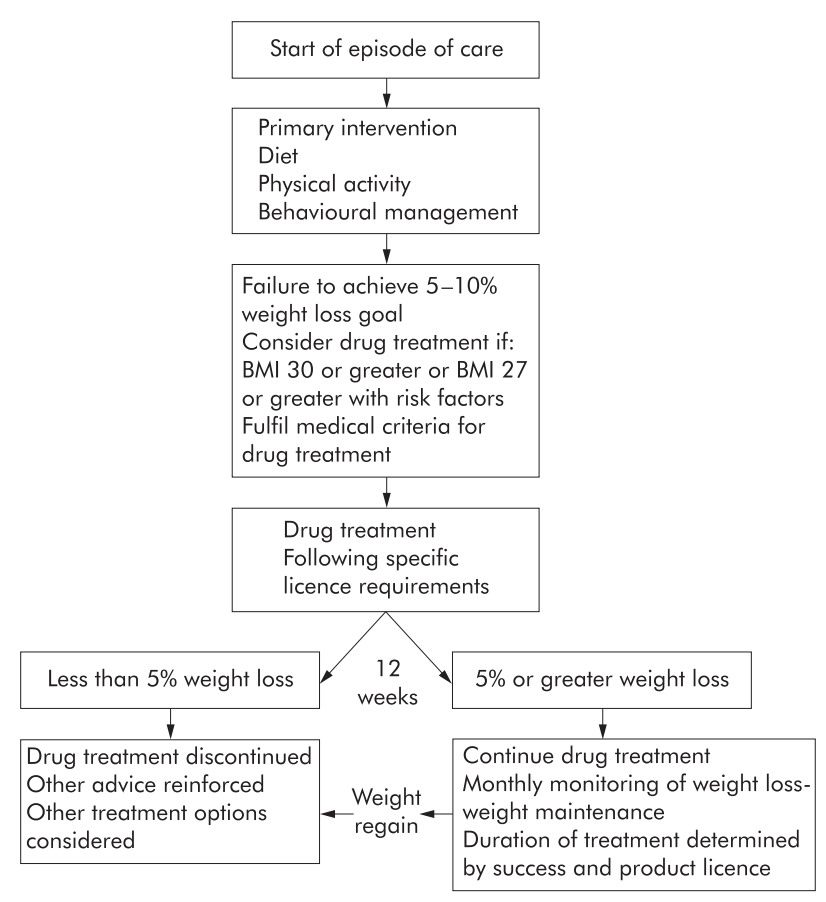

Figure 2 A management pathway for the appropriate prescription of an antiobesity drug. The firstline of treatment is dietary and physical activity interventions with some form of behaviour management. If at 12 weeks after starting such treatment, an overweight or obese patient considered at medical risk from their fatness has not achieved an appropriate weight loss goal (5-10\% of their presenting weight), then drug therapy may be considered. Drug therapy must be monitored regularly and discontinued if a patient begins to regain weight despite the drug - the duration of drug treatment should not exceed that determined by the drug's product licence (with acknowledgment to the Royal College of Physicians of London).

by the Royal College of Physicians of London about the appropriate prescribing of these drugs (fig 2). ${ }^{43}$

\section{Drugs acting on the gastrointestinal system: pancreatic lipase inhibitors}

Orlistat inhibits pancreatic and gastric lipase thereby decreasing ingested triglyceride hydrolysis. It produces a dose dependent reduction in dietary fat absorption: weight loss in obese subjects largely results from reduction in fat intake to avoid gastrointestinal effects, including steatorrhoea. Vitamin supplementation (especially of vitamin D) may be considered if there is concern about deficiency of fat soluble vitamins. Orlistat is not licensed for use longer than two years because there is insufficient experience beyond this period. However, on stopping orlistat, there may be a gradual reversal of weight loss.

\section{Centrally acting antiobesity drugs}

Sibutramine promotes a sense of satiety through its central action as a serotonin and norepinephrine reuptake inhibitor. In addition, it may mitigate against the fall in thermogenesis through stimulation of peripheral norepinephrine receptors. It is used in the adjunctive management of obesity in individuals with a BMI of $30 \mathrm{~kg} / \mathrm{m}^{2}$ or more (and no associated comorbidity) or in individuals with a BMI of $27 \mathrm{~kg} / \mathrm{m}^{2}$ or more in the presence of other risk factors such as type 2 diabetes or hypercholesterolaemia. Sibutramine is not licensed for use longer than one year, and on discontinuing treatment there may be a reversal of weight loss.

\section{Efficacy of current pharmacological treatments for obesity}

Randomised controlled trials of the use of orlistat and sibutramine suggest that approximately $60 \%$ of treated patients will achieve and maintain a 5\% loss from their starting weight after 12 months of treatment, and $40 \%$ of patients will experience a $10 \%$ weight loss. The use of both drugs is limited by side effects. Any deviation from a low fat diet while taking orlistat results in unpleasant and often explosive diarrhoea. The use of sibutramine in clinical practice may be accompanied by an elevation in blood pressure that requires close monitoring. The use of antiobesity drugs must be for those patients at medical risk from their obesity where primary intervention by dietary restriction and increased physical activity has failed to achieve medically beneficial weight loss. The National Institute for Clinical excellence (NICE) has provided guidance for the use of orlistat and sibutramine. ${ }^{44} 45$

\section{Potential future drugs for the treatment of obesity Leptin}

The initial hopes that leptin would provide a therapeutic breakthrough for the treatment of human obesity have not been realised. Leptin treatment in patients with the rare syndrome of congenital leptin deficiency does result in substantial weight reduction-restoration of plasma leptin concentrations results in progressive weight loss over a two year period. ${ }^{46}$ By contrast, initial trials of leptin in obese patients (without leptin deficiency) showed no benefit in terms of weight reduction unless substantial doses were given. Even high doses, which produced plasma concentrations $\sim 20$ times above placebo treatment baseline, resulted in variable weight loss with any benefit being countered by the requirement to give the large volume of drug by subcutaneous injection. ${ }^{47}$

The fact that some obese individuals overeat despite high plasma leptin concentrations suggests leptin resistance. This resistance could be due to a rate limiting transport step for the entry of leptin into the brain or impaired receptor signalling. There is waning interest in the development of leptin analogues and leptin receptor agonists as possible alternative treatments for obesity.

\section{GLP- 1 and exendin}

Several studies in patients with type 2 diabetes have demonstrated that GLP-1, whether administered intravenously or by subcutaneous injection, normalises both fasting and postprandial glucose levels, mainly by delaying gastric emptying and inhibition of glucagon secretion. ${ }^{48}$ In addition, the glucose lowering effect of GLP-1 is enhanced by the sulphonylurea glibenclamide in patients previously resistant to oral antidiabetic agent therapy. ${ }^{49}$ This is consistent with GLP-1 promoting islet cell function via mechanisms that are distinct from those of existing insulin secretogogues. Unfortunately, GLP-1 has a very short half life and therefore sustained response requires continuous intravenous infusion and has led to the interest in isolating more stable GLP-l that are resistant to enzymatic degradation.

Exendin-4 is a 39 amino acid peptide isolated from the salivary secretion of Heloderma suspectum, a venomous lizard found in desert regions in south western USA and 
northern Mexico. Exendin-4 shows approximately 53\% amino acid identity to human GLP-1 but differences in the penultimate amino acid sequence render it more resistant to degradation.

Exendin-4 mimics the majority of the peripheral actions of GLP-1 and reduces both gastric emptying and glucagon secretion. Various animal studies have shown that it induces satiety, weight loss, and improved insulin sensitivity, all of which result in favourable effects on blood glucose levels. The satiety effects of exendin-4 have been demonstrated in healthy subjects with associated reductions in fasting and postprandial glucose levels. ${ }^{50}$ These preliminary findings suggest that exendin-4 may have a use in the treatment of obese patients with type 2 diabetes.

\section{Peptide $\mathrm{YY}_{3-36}$}

A recent study has shown that a single infusion of PYY, as compared with an equivalent infusion of saline, reduced appetite and food consumption by approximately $30 \%$ at a self-selection buffet lunch provided two hours after the infusion. ${ }^{51}$ In obese subjects, the endogenous postprandial PYY response was diminished compared with lean subjects even though obese subjects consumed a greater number of calories. PYY infusion reduced hunger in both the obese and lean groups and had no effect on subjects' reports of palatability of the meals or their feelings of nausea. These very preliminary findings suggest that PYY may prevent counterregulatory mechanisms from overriding stimulation of anorexigenic pathways. However, the findings to date relate to a single infusion of PYY and its immediate actionthere is no published information about its longer term use.

\section{Other agents}

There is considerable interest in the development of novel agents for the treatment of obesity. The most effective option might circumvent leptin resistance by targeting an appetite regulating pathway downstream from the leptin receptor activation such as NPY, $\alpha$-MSH, or the melanocortin receptor.

The potency and efficacy of exogenous NPY as an appetite stimulant after its acute administration, and its persistent effects during chronic administration in rodents, suggest that NPY may play an essential role in short and long term regulation of energy balance. Studies suggest that the $\mathrm{NPY}_{5}$ receptor may be the molecular target for appetite stimulation. There is interest in developing selective $\mathrm{NPY}_{5}$ antagonists.

Clinical observation has confirmed that the melanocortin meditated pathway also plays an important role in the regulation of body weight in humans. Human POMC mutations and various mutations in the gene encoding the MC4 receptor have been identified as being associated with an obese phenotype. Such mutations appear to be the most common monogenic form of human obesity. This suggests that agonists to the MC4 receptor could prove effective in enhancing weight reduction.

Finally, there is renewed interest in the administration of synthetically derived cholecystokinin as a gut signal. Cholecystokinin has anorectic effects through a central action in addition to alterations in gastric motility.

\section{SURGICAL TREATMENT OF OBESITY}

There are three operative procedures currently used for the surgical treatment of obesity: gastric restriction and gastric bypass operations, and biliopancreatic diversion (fig 3).
Gastric restriction involves the creation of a small capacity compartment $(<20 \mathrm{ml})$ by either a combination of vertical stapling and a constrictive band opening or a circum-gastric band pinching off a small proximal pouch. A modification of the latter procedure is an inflatable circumgastric band attached to a subcutaneous reservoir which allows access by a hypodermic syringe to inject or withdraw fluid, thereby tightening or enlarging the band width.

Gastric bypass is performed by stapling shut a $<20 \mathrm{ml}$ vertically oriented pouch and connecting this pouch to the jejunum transected $50 \mathrm{~cm}$ from the ligament of Treitz (Rouxen-Y gastric bypass). Published evidence confirms that this procedure produces greater weight loss but is accompanied by more frequent adverse effects, including "dumping". ${ }^{52}$

Biliopancreatic diversion includes gastric resection and diversion of the biliopancreatic juice to the terminal ileum to reduce the absorption of nutrients. In this operation, an entero-entero anastomosis is performed between the proximal limb of the transected jejunum and ileum, 50-100 cm proximal to the ileocaecal valve. A more recent modification maintains the pylorus and a portion of the duodenum ("duodenal switch"), which improves protein absorption and results in fewer side effects (fig 3).

Most antiobesity surgical procedures have been successfully performed laparoscopically which reduces the requirement for analgesia and facilitates prompt postoperative mobilisation.

\section{Efficacy of surgical treatment}

Surgery is usually successful in inducing substantial weight loss in the majority of obese patients. This is achieved primarily by a necessary reduction in calorie intake. It is of interest that gastric bypass surgery has been shown to be associated with low ghrelin levels, which may contribute to the reduced appetite associated with the procedure. PYY levels have been shown to be elevated after jejunoileal bypass surgery, suggesting that increased PYY levels may also contribute to the reduced appetite and food intake observed in some patients.

\section{Gastric restriction}

Gastric restriction operations require strict dietary compliance because an intake of high caloric liquids or soft foods are not inhibited by the narrow outlet and may explain failure to lose weight. The advantage of these techniques is their relative simplicity with no anastomoses or bypass of any part of the bowel. As a consequence, operative mortality is very low and longer term nutritional deficiencies unlikely. The reported excess weight loss after 3-5 years is $40-60 \%$ but there is a slow regain thereafter. Some patient do not lose weight.

\section{Gastric bypass}

Gastric bypass operations generally achieve an excess weight loss of 49-62\% maintained over a 5-14 year period. The nature of the operation is more complicated and operative mortality approximately $1 \%$. This operative procedure can be associated with nutritional deficiency although this is unusual when detailed dietary advice is reinforced with dietary follow up.

\section{Biliopancreatic division}

Biliopancreatic division achieves up to $78 \%$ excess weight loss at 18 years. Again, operative mortality is $1 \%$ but nutritional 

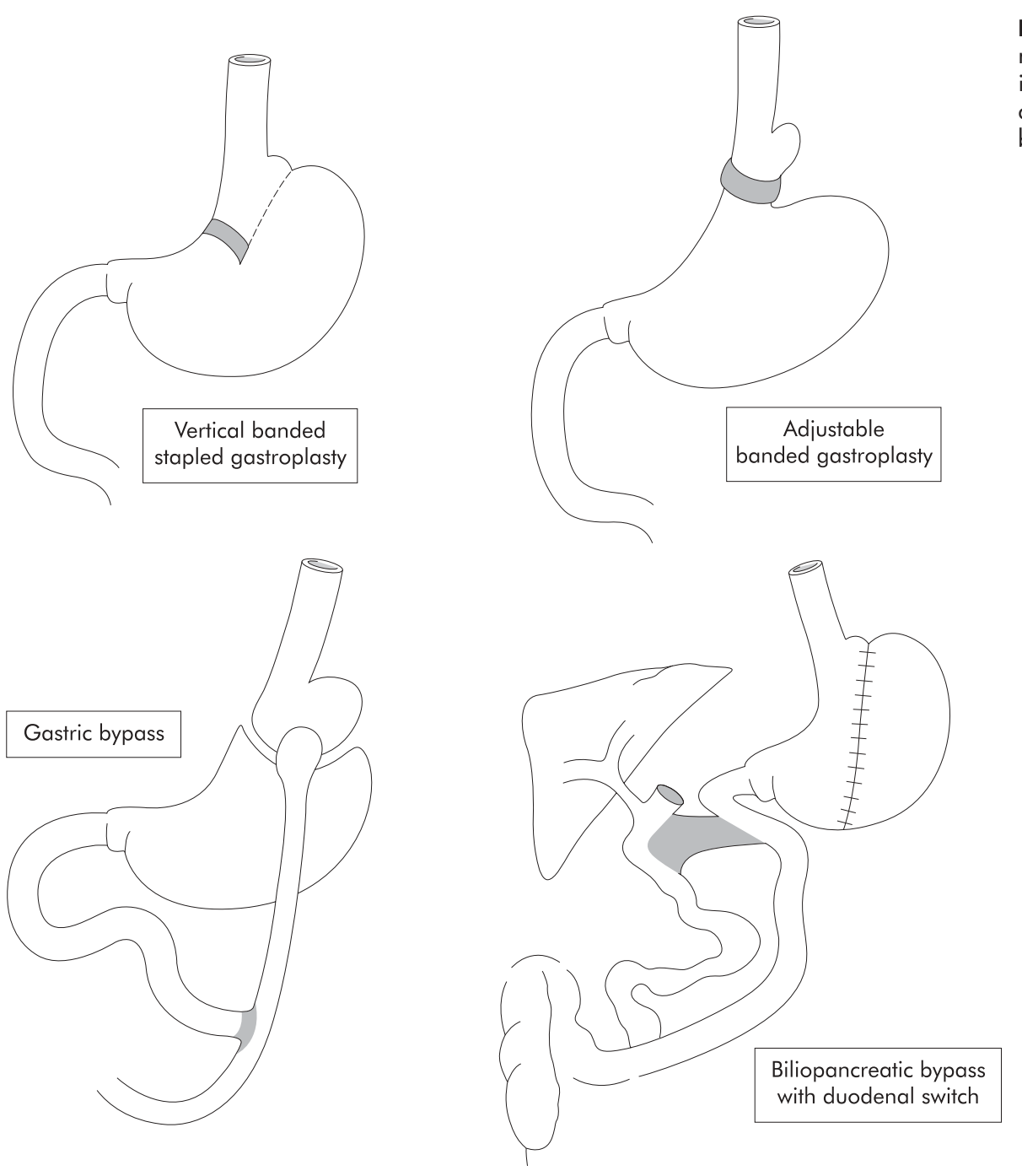

Figure 3 Three surgical procedures for reducing gastric volume as a method for inducing weight loss: gastric restriction and gastric bypass operations, and biliopancreatic diversion.

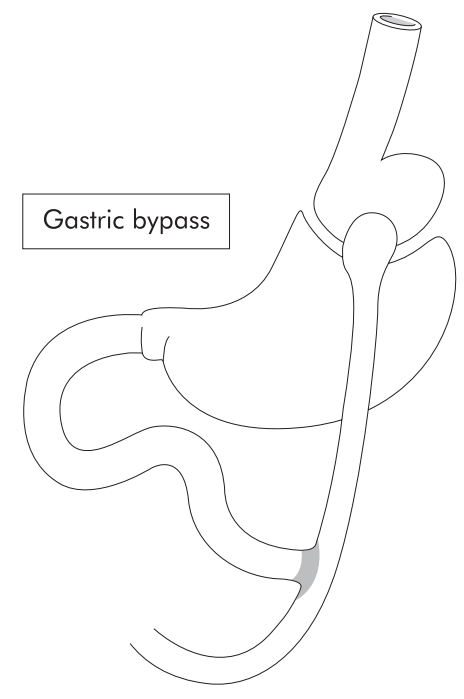

deficiencies are relatively common $(5-40 \%$ of patients for the longer term). In addition, alterations in bowel movements are frequent with 3-5 motions, commonly offensive, occurring each day.

\section{Long term outcome following obesity surgery}

The Swedish Obese Subjects (SOS) study is a prospective intervention study that has evaluated the medical outcomes from obesity surgery over a 10 year period. The reported outcome to date after eight years has shown a weight loss of $16.3 \mathrm{~kg}$ compared with no loss in a medically treated control group..$^{53}$ The unadjusted prevalence of diabetes among the controls followed for eight years increased from $7.8 \%$ to $24.9 \%$ while in the surgically treated group the prevalence rate was stable at $10.5 \%$. The course of blood pressure showed a different pattern with an initial significant fall in blood pressure in the surgically treated group associated with rapid weight loss. In spite of continuing weight loss during the next six years, the reduction in diastolic blood pressure ceased and was accompanied by a small rise in systolic blood pressure. As a result, there was no difference observed in systolic blood pressure between the two groups at eight years; diastolic pressure was $2.5 \mathrm{~mm} \mathrm{Hg}$ higher in the surgically treated group compared with controls, despite the weight loss. Furthermore, the initial improvement in all parameters of serum lipid profiles was not sustained in the surgically treated group at eight years-although HDL cholesterol levels increased, there was no change in total cholesterol from preoperative values. $^{54}$

A review of evidence from randomised controlled trials confirms that surgery for obesity is an option for carefully selected patients with clinically severe obesity (BMI $\geqslant 40$ or BMI $\geqslant 35$ with comorbid conditions) when less invasive methods of weight loss have failed and the patient is at high risk for obesity associated morbidity and mortality, most particularly diabetes. NICE has issued guidance about surgical treatment for morbidly obese patients and confirms that the nature of the surgical procedures necessitate long term hospital follow up for such patients. ${ }^{52}$ Table 2 outlines the selection criteria in accordance with NICE guidelines for patients considered suitable for surgical intervention for their obesity.

\section{Weight maintenance}

Obesity results in most patients not from an inability to lose weight but a profound difficulty in maintaining a lowered weight. A programme to enable the individual to maintain their lowered weight must follow any successful weight loss. Published evidence suggests that a combination of sensible eating, physical activity, and reinforcement of behavioural 
Table 2 Criteria of suitability for obesity surgery (see Heymsfield and colleagues ${ }^{47}$ )

- Only considered in those receiving intensive management in specialist hospital obesity clinic.

- All appropriate and available non-surgical measures attempted but failed to maintain weight loss.

- Aged 18 years or above.

- $\mathrm{BMI} \geqslant 40 \mathrm{~kg} / \mathrm{m}^{2}$ or BMI $35-40 \mathrm{~kg} / \mathrm{m}^{2}$ plus comorbidities that would benefit from weight loss.

- Established complications from obesity or at risk of developing complications.

- Understand the medical need for weight loss and mechanisms of surgical operation.

- Understand the potential risks and benefits of surgery.

- Have realistic expectations of long term outcome from surgery.

- No past history of significant psychiatric illness including eating disorders such as bulimia nervosa.

No history of alcoholism.

- Reliable appointment keeping and ability to follow medical and dietetic advice.

- No significant medical complication that contraindicates general anaesthesia or surgery

BMI, body mass index.

methods are the most successful for the longer term. Clinical care can reinforce their importance but the ultimate responsibility for following such advice must lie with the patient, and the duration of follow up tailored accordingly.

\section{CONCLUSIONS}

A better understanding of the mechanisms of appetite regulation and the application of this knowledge as part of evidence based interventions are leading to a more coherent approach to obesity treatment. Nevertheless, biological mechanisms have evolved to prevent weight loss, rather than excessive adiposity, and thus the likelihood of a pharmacological solution or "magic bullet" is very small. Investigations of neuroendocrine targets for appetite suppression in animal models suggest redundancy in such systems which are likely to make the development of effective agents based on single receptors impractical. Moreover, the progressive rise in the prevalence of obesity will mean that inevitably only a relatively small proportion of patients will be treated by intensive behaviour therapy, long term drug treatment, or surgery. The only effective method to manage obesity for the longer term will be its prevention.

\section{Summary}

- Obesity results from an imbalance between energy consumed relative to energy expended.

- Changes in the environment with increasing availability of food and decreasing physical activity are responsible for the substantial increase in prevalence of obesity.

- Reducing fat intake as part of dietary treatment is an effective means of reducing energy intake and reducing the risk of associated chronic diseases.

- Physical activity plays a critical role in the prevention of weight gain, the maintenance of reduced weight, and associated risk reduction.

- A behavioural approach to helping patients change their eating and activity behaviour is important in encouraging longer term changes in diet and physical activity.

- A better understanding of mechanisms regulating appetite provides an opportunity to identify potential targets for future drug treatments.

- Current drug therapies are useful adjuncts in those patients at risk from overweight and obesity where primary intervention with lifestyle modification has not resulted in weight loss sufficient to reduce medical risk.

- Surgical treatment should be considered as treatment for selected patients at considerable medical risk from morbid obesity.

\section{Authors' affiliations}

P G Kopelman, Barts and the London, Queen Mary's School of Medicine and Dentistry, University of London, London, UK

C Grace, Department of Diabetes and Metabolic Medicine, Royal London Hospital, London, UK

\section{REFERENCES}

1 Joint Health Survey (on behalf of the Department of Health). Health Survey for England, 2000. London: The Stationery Office, 2001

2 Kopelman PG. Obesity as a medical problem. Nature 2000;404:635-43.

3 Lew EA. Mortality and weight: insured lives and the American Cancer Study. Ann Int Med 1985;103:1024-9.

4 Hubert HB, Feinleib M, McNamara PM, et al. Obesity as an independent risk factor for cardiovascular disease: a 26 -year follow-up of participants in the Framingham heart study. Circulation 1983;67:968-77.

5 Reaven GM. Banting Lecture 1988. Role of insulin in human disease. Diabetes 1988;37:1595-607.

6 Poppitt SD, Swann D, Black AE, et al. Assessment of selective under-reporting of food intake by both obese and non-obese women in metabolic facility. Int J Obes 1998;22:303-11.

7 Young LR, Nestle M. The contribution of expanding portion sizes in the US obesity epidemic. Am J Public Health 2002;92:246-9.

8 Rissanen AM, Heliovaara M, Knekt $P$, et al. Determinants of weight gain and overweight in adult Finns. Eur J Clin Nutr 1991;45:419-30.

9 Gortmaker SL, Must A, Sobol AM, et al. Television viewing as a cause of increasing obesity among children in the United States. Arch Pediatr Adolesc Med 1996;150:356-62.

10 OPCS (Office of Population Censuses and Surveys). General Household Survey. London: HMSO, 1994.

11 Prentice AM, Jebb SA. Obesity in Britain: gluttony or sloth? $\mathrm{Br}$ Med J 1995:311:437-9.

12 Zhang $Y$, Proenca R, Maffei $M$, et al. Positional cloning of the mouse obese gene and its human homologue. Nature 1994;372:425-32.

13 Montague CT, Faroogi IS, Whitehead JP, et al. Congenital leptin deficiency is associated with severe early onset obesity in humans. Nature 1997;387:903-9.

14 Considine RV, Sinha MK, Heiman ML, et al. Serum immunoreactive-leptin concentrations in normal weight and obese humans. N Engl J Med 1996:334:292-5.

15 Mantzoros CS. The role of leptin in human obesity and disease: A review of the evidence. Ann Intern Med 1999;130:671-80.

16 Schwartz MW, Woods SC, Porte D ir, et al. Central nervous system control of food intake. Nature 2000;404:661-71.

17 Ahima RS, Osei SY. Neuroendocrine regulation of appetite and energy balance. Curr Opin Endocrinol Diabetes 2002;9:215-23.

18 Gutniak M, Orskov C, Holst JJ, et al. Antidiabetogenic effect of glucagon-like peptide-1 (7-36) amide in normal subjects and patients with diabetes mellitus. N Engl J Med 1992;326:1316-22.

19 Turton MD, O'Shea D, Gunn I, et al. A role of glucagon-like peptide-1 in the central regulation of feeding. Nature 1996;379:69-72.

20 Batterham RL, Cowley MA, Small CJ, et al. Gut hormone PYY (3-36) physiologically inhibits food intake. Nature 2002;418:650-4

21 Horvath TL, Diano S, Heiman ML. Ghrelin induces adiposity in rodents. Nature 2001;407:908-13.

22 National Institutes of Health. National Heart, Lung and Blood Institute. Clinical guidelines on the identification, evaluation and treatment of overweight and obesity in adults. The evidence report. Bethesda: NIH, 1998.

23 Department of Health. Dietary reference values for food energy and nutrients for the United Kingdom. (Report on health and social subjects; 41). London: HMSO, 1991

24 Bray GA, Popkin BM. Dietary fat intake does affect obesity! Am J Clin Nutr 1998;68:1157-73. 
25 Astrup A, Grunwald GK, Melanson EL, et al. The role of low-fat diets in body weight control: a meta-analysis of ad libitum dietary intervention studies. Int J Obes Relat Metab Disord 2000;24:1545-52.

26 Yu-Poth S, Zhao G, Etherton T, et al. Effects of the National Cholesterol Education Program's Step and Step II dietary intervention programs on cardiovascular disease risk factors: a meta-analysis. Am J Clin Nutr 1999;69:632-46

27 Mulvihill C, Quigley R. The management of obesity and overweight: an analysis of reviews of diet, physical activity and behavioural approaches. Evidence briefing. London: Health Development Agency, 2003.

28 Knowler WC, Barett-Connor PH, Fowler SE, et al. Reduction in the incidence of type 2 diabetes with lifestyle intervention or metformin. Diabetes Prevention Programme Research Group. N Engl J Med 2002;346:393-403.

29 Tuomilehto J, Lindstrom J, Eriksson JG, et al. Prevention of type 2 diabetes mellitus by changes in lifestyle among subjects with impaired glucose tolerance. N Engl J Med 2001;344:1343-50.

30 Frost G, Masters K, King C, et al. A new method of energy prescription to improve weight loss. J Hum Nutr Diet 1991;4:369-73.

31 Scottish Intercollegiate Guidelines Network (SIGN). Obesity in Scotland: Integrating Prevention with Weight Management. A National Clinical Guideline Recommended for Use in Scotland by the Scottish Intercollegiate Guidelines Network (pilot edn). SIGN: Edinburgh, 1996.

32 Heymsfield SB, van Mierlo CAJ, van der Knaap HCM, et al. Weight management using a meal replacement strategy: meta and pooling analysis from six studies. Int J Obesity 2003;27:537-49.

33 Wadden TA, Foster GD, Letizia KA. One year behavioural treatment of obesity: comparison of moderate and severe caloric restriction and the effects of weight maintenance therapy. J Consult Clin Psychol 1994;62:165-71.

34 Raben A. Should obese patients be counselled to follow a low-glycaemic index diet? No. Obes Rev 2002;3:245-56.

35 Frost G, Leeds AA, Dore CJ, et al. Glycaemic index as a determinant of serum HDL-cholesterol concentration. Lancet 1999;353:1045-8.

36 St Jeor ST, Howard BV, Prewitt TE, et al. Nutrition Committee of the Council on Nutrition, Physical Activity, and Metabolism of the American Heart Association. Dietary protein and weight reduction: a statement for healthcare professionals from the Nutrition Committee of the Council on Nutrition, Physical Activity, and Metabolism of the American Heart Association, Circulation 2001:9:1869-74.

37 Samaha FF, lqbal N, Seshadri P, et al. A low-carbohydrate as compared to a low-fat diet in severe obesity. N Engl J Med 2003;348:2074-81.
38 Foster G, Wyatt HR, Hill JO, et al. A randomised trial of a low carbohydrate diet for obesity. N Engl J Med, 2003;348, 21:2082-90.

39 Saris WHM. Physiological aspects of exercise in weight cycling. Am J Clin Nutr 1989;49:1099-104.

40 Ravussin E, Bogardus C. A brief overview of human energy metabolism and its relationship to essential obesity. Am J Clin Nutr 1992:55:242S-5S.

41 Saris WH, Blair SN, van Baak MA, et al. How much physical activity is enough to prevent unhealthy weight gain? Outcome of IASO $1^{\text {st }}$ Stock conference and consensus statement. Obes Rev 2003;4:101-14.

42 Thorogood M, Hillsdon M, Summerbell C. Changing behaviour. Clin Evidence 2002;8:37-59.

43 Anti-obesity drugs: guidance on appropriate prescribing and management. London: Royal College of Physicians of London, 2003

44 NICE. Guidance on the use of orlistat for the treatment of obesity in adults: NICE technology appraisal guidance No 22. London: NICE, 2001.

45 NICE. Guidance on the use of sibutramine for the treatment of obesity in adults. NICE technology appraisal guidance No 31. London: NICE, 2002

46 Farooki LS, Jebb S, Cook G, et al. Treatment of congenital leptin deficiency in man, 8th International Congress on Obesity Abstracts Book. Paris: Convergences ICO, 1998, 7 (abstract).

47 Heymsfield SB, Grenberg AS, Fujioka K, et al. Recombinant leptin for weight loss in obese and lean adults. JAMA, 1999;282:1568-75.

48 Nauck MA, Wollschlager D, Werner J, et al. Effects of subcutaneous glucagon like peptide-1 in patients with NIDDM. Diabetologia 1996;39:1546-53.

49 Gutniak MK, Juntti-Berggren L, Hellstrom PM. Glucagon like peptide-1 enhances the insulinotrophic effect of glibenclamide in NIDDM patients and in the perfused rat pancreas. Diabetes Care 1996;19:857-63.

50 Edwards CM, Stanley SA, Davis R, et al. Exendin-4 reduces fasting and postprandial glucose and decreases energy intake in healthy volunteers. Am J Physiol Endocrinol Metab 2001;281:E155-61.

51 Batterham RL, Cohen MA, Ellis SM, et al. Inhibition of food intake in obese subjects by peptide $\mathrm{YY}_{3-36}$. N Engl J Med 2003;349:941-8.

52 NICE. Full guidance on the use of surgery to aid weight reduction for people with morbid obesity. NICE technology appraisal guidance No 46. London: NICE, 2002.

53 Sjostrom CD, Peltonen $M$, Wedel $H$, et al. Differentiated long-term effects of intentional weight loss on diabetes and hypertension. Hypertension 2000;36:20-5.

54 Sjostrom L. Swedish obese subjects, SOS. In: Bjorntorp P, eds. International textbook of obesity. Chichester: John Wiley and Sons, 2001, 519-33.. 